$\begin{array}{ll}\text { Research Square } & \text { Preprints are preliminary reports that have not undergone peer review. } \\ \text { They should not be considered conclusive, used to inform clinical practice, } \\ \text { or referenced by the media as validated information. }\end{array}$

\title{
A maternal GOT1 novel mutation associated with early-onset severe preeclampsia identified by whole-exome sequencing
}

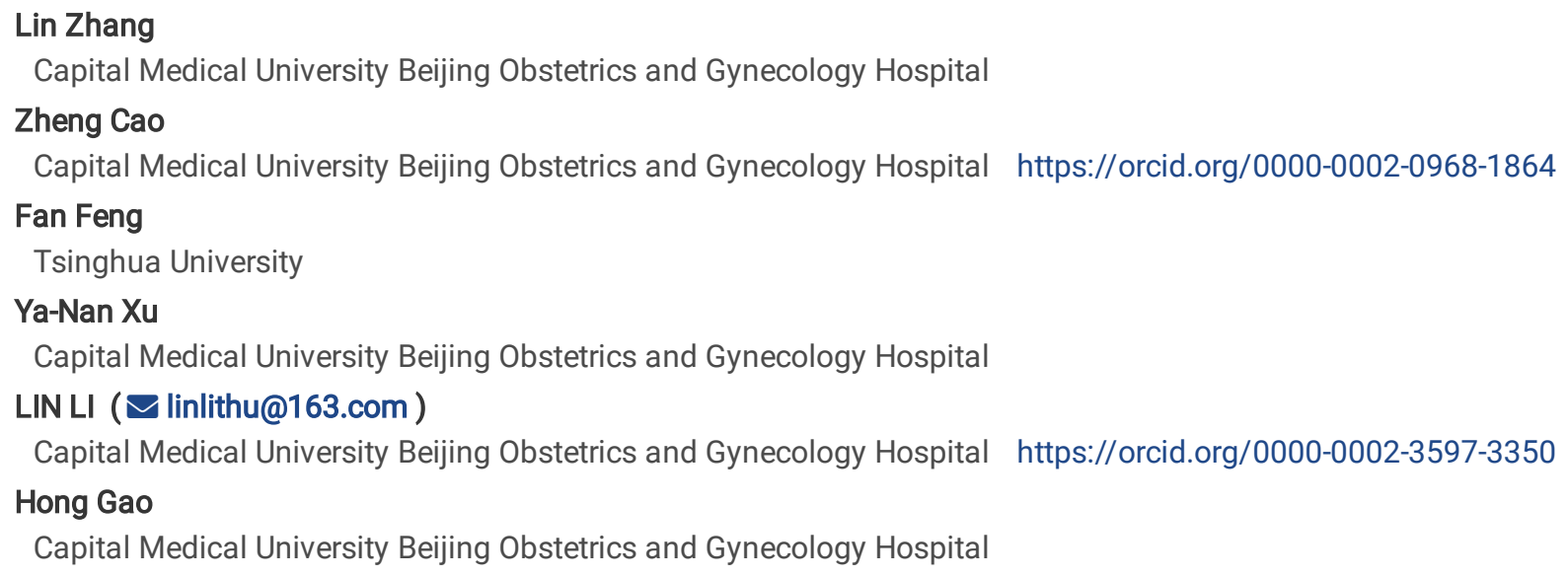

Version of Record: A version of this preprint was published at BMC Medical Genetics on March 6th, 2020. See the published version at https://doi.org/10.1186/s12881-020-0989-2. 


\section{Abstract}

Purpose This study wants to know the genetic cause of preeclampsia (PE) which is a leading cause of maternal and perinatal death, but the underlying molecular mechanisms that cause PE remain poorly understood. Many single nucleotide polymorphisms have been identified by genome-wide association studies and were found to be associated with PE; however, few studies have used whole-exome sequencing (WES) to identify PE mutations. Methods Five patients with severe early-onset preeclampsia (EOPE) were recruited, and WES was performed on each patient. Sanger sequencing was used to confirm the potential causative genetic mutation. Results After a stringent bioinformatics analysis, a rare mutation in the GOT1 gene, c.44C>G:p.P15R, was found in one patient. Bioinformatics analysis showed that the mutation site is highly conserved across several species and was predicted to be a pathogenic mutation according to several online mutational function prediction software packages. Further structural biology homology modeling suggested that P15R would change the electric environment of enzymatic center, and might affect the binding affinity of substrate or product. Conclusion We demonstrated for the first time that the mutation in GOT1 may be associated with EOPE, the results of this study provide researchers and clinicians with a better understanding of the molecular mechanisms that underlie maternal severe EOPE.

\section{Introduction}

Preeclampsia (PE) is a pregnancy-specific multi-systemic syndrome that affects several organs, including the kidneys, liver, and brain, and is a leading cause of maternal and perinatal morbidity and mortality. PE affects approximately $5 \%$ of pregnancies and is characterized by new-onset hypertension and proteinuria at $\geq 20$ weeks of gestation [1]. The main manifestation of preeclampsia is proteinuria, as well as thrombocytopenia, impaired liver function, new development of renal insufficiency, pulmonary edema, or new-onset cerebral or visual disturbances. The placenta plays a key role in the development of this disorder. The pathogenic mechanism of PE includes defective deep placentation, oxidative and endoplasmic reticulum stress, autoantibodies to type- 1 angiotensin II receptor, platelet and thrombin activation, intravascular inflammation, endothelial dysfunction, presence of an antiangiogenic state, and maternal angiogenic imbalances caused by placental antiangiogenic factors [2-8].

Genetic alteration is one of the main causes of PE [9-11], but the exact underlying molecular mechanism remains unclear. Maternal or fetal sequence variants located in or near some genes, such as FLT1 [12, 13], PLEKHG1 [14], APOL1 [15], ERAP2 [16, 17], WNK1, and AKR1C3 [18], CYP2D6 and CYP2C9 [19], were shown to be associated with PE. Genome-wide association studies and Sanger sequencing are frequently used in the genetic analysis of $\mathrm{PE}$, while high-throughput sequencing technology such as whole-exome sequencing (WES) is rarely used to find the genetic cause of $\mathrm{PE}$.

Here, we recruited 5 unrelated patients with severe EOPE and performed WES for each patient. Sequence variants were filtered according to standard procedures. A novel mutation in GOT1 was identified in one patient (P1), and is considered to be a potentially pathogenic mutation associated with $\mathrm{PE}$, according to in silico analysis and structural biology predictions.

\section{Materials And Methods}

\section{Subjects}

PE can be characterized into 2 different disease entities: early-onset PE (EOPE, corresponding to preeclampsia registered at $<34$ weeks) and late-onset PE (LOPE corresponding to preeclampsia registered at $>34$ weeks). They are associated with different fetal and maternal effects, heritability, biochemical markers, and clinical symptoms [20]. We chose patients diagnosed with EOPE at the Beijing Obstetrics and Gynecology Hospital between January 2018 and June 2018. The diagnostic criteria of severe PE include: blood pressure of $160 / 110 \mathrm{mmHg}$ or higher; thrombocytopenia (platelet count below 100,000/ $\mu \mathrm{L}$ ); impaired liver function as indicated by abnormally elevated blood concentration of liver enzymes; severe persistent right upper abdominal pain; progressive renal insufficiency (serum creatinine concentration above $1.1 \mathrm{mg} / \mathrm{dL}$ or doubling of serum creatinine concentration); pulmonary edema; and cerebral or visual disturbances. Women with high risk factors [21] for PE, such as those with chronic hypertension, pre-gestational diabetes, maternal body mass index (BMI) $>30 \mathrm{~kg} / \mathrm{m}^{2}$, antiphospholipid syndrome/SLE, or who had received assisted reproduction, were excluded from our study. However, we did not rule out women with a prior history of pre-eclampsia. Using the above criteria, we identified 5 patients with severe EOPE (Table 1).

All procedures involving human participants were performed in accordance with the ethical standards of the Ethics Committee of Beijing Obstetrics and Gynecology Hospital and with the 1964 Helsinki declaration and its later amendments. Written informed consent was obtained from each patient.

\section{Detailed clinical information for P1}

Page $2 / 10$ 
Patient No. 1 (P1) is a 35-year-old woman, body weight $62.5 \mathrm{~kg}$, with a BMI of $22.9 \mathrm{~kg} / \mathrm{m}^{2}$ prior to pregnancy. Six years ago, she delivered a baby girl by cesarean section because the umbilical cord was wrapped around the fetus's neck. During the same period, she was diagnosed with severe PE (gestational age of onset is unknown), and her blood pressure returned to normal postpartum. She refused to provide a personal medical history or a family history. The patient's blood pressure rose to 140/90 mmHg at 25 weeks of gestation, and an ultrasound examination showed that the fetus was small. She began to experience limb edema at 26 weeks of gestation but was not treated at this time. At 28 weeks of gestation, her blood pressure rose to 197/114 mmHg and urinary protein appeared as $3+$, but she made no complaints. When the patient visited our hospital at that time, her body weight was $80 \mathrm{~kg}$, and an ultrasound test showed that umbilical cord blood flow was S/D 4.8 and fetal growth was restricted. Her urinary protein went up to $4+$, and $24 \mathrm{~h}$ urine protein was 7431.2 mg. The highest level of serum creatine was $85.6 \mu \mathrm{mol} / \mathrm{L}$, blood urea nitrogen was $10.56 \mathrm{mmol} / \mathrm{L}$, uric acid was $472.9 \mathrm{mmol} / \mathrm{L}$, with no detected anemia or thrombocytopenia. The lowest level of albumin was $22.6 \mathrm{~g} / \mathrm{L}$. As for liver damage, the highest level of alanine aminotransferase was $52.5 \mathrm{U} / \mathrm{L}$, aspartate aminotransferase was $41.6 \mathrm{U} / \mathrm{L}$, and lactate dehydrogenase was $332 \mathrm{U} / \mathrm{L}$. The highest level of d-dimer was 10.87 $\mathrm{mg} / \mathrm{L}$. Echocardiography showed no abnormalities. Both antinuclear antibodies, antibody spectrum, and cardiolipin antibody were all negative. After administering drugs and inducing labor, P1 gave birth to a stillborn child. She recovered well and was released from the hospital 10 days later.

\section{Detailed clinical information for P2}

Patient No. 2 (P2) is a 27-year-old woman who was gravida 1, para 0, and her BMI was $22.1 \mathrm{~kg} / \mathrm{m}^{2}$ prior to pregnancy. She denied personal medical history or family history. Down screening showed high risk of open neural tube malformation at 13 weeks of gestation. The patient's presented with high blood pressure of $178 / 120 \mathrm{mmHg}$ during regular prenatal examination at 27 weeks of gestation, with complaint about headache, dizziness and edema of lower extremity for half a month, and nausea for a week or so. The serum creatine went up to $100.9 \mu \mathrm{mol} / \mathrm{L}$ within the next week. Her 24-hour urine protein quantification reached $7212.5 \mathrm{mg} / 24 \mathrm{~h}$. No anemia or thrombocytopenia was found. Lupus anticoagulant and anticardiolipin antibody tests were both negative. The patient received antispasmodic treatment with magnesium sulfate, and antihypertensive therapy. However, the patient developed oliguria, pleural effusion and peritoneal effusion邓then she underwent cesarean section. The weight of neonatal was $885 \mathrm{~g}$. Apgar score after birth was 9'-9'-9'. After the operation, the serum creatine went up to $121.2 \mu \mathrm{mol} / \mathrm{L}$, with the serum urea nitrogen $11.76 \mathrm{mmol} / \mathrm{L}$, and the uric acid $692.3 \mathrm{mmol} / \mathrm{L}$, and the minimum level of albumin was $24.0 \mathrm{~g} / \mathrm{L}$. The patient continued to receive antihypertensive, diuretic and prophylactic anticoagulant therapy. Finally, the patient recovered well and left hospital 6 days after the operation.

\section{Detailed clinical information for P3}

Patient No. 3 (P3) is a 29-year-old woman, who was gravida 1, para 0. Her BMI was $20.0 \mathrm{~kg} / \mathrm{m}^{2}$ prior to pregnancy. She denied personal medical history or a family history. The patient's presented with high blood pressure of $145 / 100 \mathrm{mmHg}$ and her urinary examination showed urinary protein (3+) during regular prenatal examination at 27 weeks of gestation. She didn't complain about headache, dizziness or edema. Her CVT indicated high peripheral resistance. Her 24-hour urine protein quantification could reach $3882.0 \mathrm{mg} / 24 \mathrm{~h}$. Lupus anticoagulant and anticardiolipin antibody tests were both negative. The patient received antispasmodic treatment with magnesium sulfate, and antihypertensive therapy. As a result of rapidly elevating blood pressure of 180/120 mmHg and upper abdominal pain, the patient underwent urgent cesarean section. The weight of neonatal was $1110 \mathrm{~g}$. Apgar score after birth was 10'-10'-10'. One day after the operation, the platelet count fell down to $66^{*} 10^{9} / \mathrm{L} \rrbracket$ and ALT level elevated to $110.6 \mathrm{IU} / \mathrm{L}, \mathrm{AST} 50.9$ IU/L『LDH 375 IU/L『Crea $90.1 \mu \mathrm{mol} / \mathrm{L} \rrbracket$ BUN 9.03 mmol/L, UA706.8 $\mu \mathrm{mol} / \mathrm{L}$. Her abdominal ultrasound showed no abnormality. She was therefore diagnosed with HELLP syndrome. The patient then received antihypertensive, diuretic and prophylactic anticoagulant therapy, and she recovered and left hospital 6 days after the operation.

\section{Detailed clinical information for P4}

Patient No. 4 (P4) is a 31-year-old woman, who was gravida 2, para 0. Her BMI was $26.8 \mathrm{~kg} / \mathrm{m}^{2}$ prior to pregnancy. And she was diagnosed with preeclampsia and intrauterine fetal death occurred at 24 weeks of gestation 3 years ago. The patient's mother had hypertension in her old age. The patient's presented with high blood pressure of 140/90 mmHg complaining about headache and dizziness for three weeks at 26 weeks of gestation. Her 24-hour urine protein quantification could reach $1300 \mathrm{mg} / 24 \mathrm{~h}$. Ultra-sound test showed fetal growth restriction. Umbilical cord blood flow showed $B=0$, and the patient received Induction of labor by amniocentesis and rivanol. After two days of operation, the blood pressure rose to $190 / 124 \mathrm{mmHg}$, and the patient complained about upper abdominal pain. Biochemical tests showed

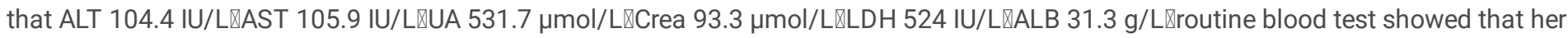
platelet account fell down to $100 * 10^{9}$ /L『the haemoglobin level was in normal range. She was diagnosed with partial HELLP syndrome. 
Lupus anticoagulant was positive, and anticardiolipin antibody tests was negative. The patient received antihypertensive, diuretic and prophylactic anticoagulant therapy, and she recovered and left hospital 6 days after the operation.

\section{Detailed clinical information for P5}

Patient No. 5 (P5) is a 31-year-old woman, who was gravida 1, para 0. Her BMI was $26.8 \mathrm{~kg} / \mathrm{m}^{2}$ prior to pregnancy. She denied personal medical history or a family history. The patient developed pruritus at 28 weeks of gestation. She presented with high blood pressure of $170 / 120 \mathrm{mmHg}$ with edema during regular prenatal examination at 30 weeks of gestation, and biochemical examinations showed that ALT

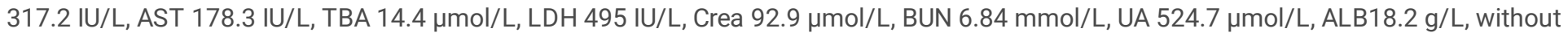
hemolysis or thrombocytopenia. Her urinary protein was $3+\bigotimes$ the 24 -hour urine protein quantification could reach $2884.8 \mathrm{mg} / 24 \mathrm{~h}$. BNP level was $515.6 \mathrm{pg} / \mathrm{ml}$. And ultrasound showed that massive ascites could be found. She was diagnosed with severe preeclampsia冈partial HELLP syndrome and intrahepatic cholestasis of pregnancy. Then she received emergency cesarean section at 31 weeks of gestation, and the weight of the newborn baby is $1355 \mathrm{~g}$. Apgar score after birth was 10'-10'-10'. After liver protecting and antihypertensive therapies, she recovered and left hospital 5 days after the operation.

\section{Whole-exome sequencing analysis}

Genomic DNA was extracted from peripheral blood using a DNeasy Blood and Tissue Kit (Qiagen, Valencia, CA, USA). Whole-exome sequencing (WES) was performed by Annoroad Gene Technology Co., Ltd, Beijing. Briefly, exomes were captured using a SureSelect Human All Exon V6 Kit (Agilent Technologies), and were sequenced using a HiSeq X10 Sequencer (IIlumina). Raw Reads were mapped against the human reference genome hg19 using Burrows-Wheeler Aligner (BWA). Single nucleotide variants (SNV) were identified by SAMTools and Genome Analysis Toolkit (GATK) software, and ANNOVAR was used for SNV functional annotation and filtering. Variants fulfilling the following criteria were retained: (i) missense, nonsense, frame-shift, or splice site variants; (ii) absent from the Exome Aggregation Consortium database (ExAC, http://exac.broadinstitute.org/), Genome Aggregation Database (gnomAD, http://gnomad.broadinstitute.org/), 1000 Genomes (http://browser.1000genomes.org/index.html), ESP6500 (http://evs.gs.washington.edu/EVS/), and our inhouse database.

\section{Sanger sequencing validation}

The whole-exome sequencing results were validated using Sanger sequencing. For the GOT1 (c.44C>G:p.P15R) variant, forward (5'ATTGGTTAATCGCGTTGCCAA-3') and reverse (5'-CCACACCTGCATCTGTAAAATGG-3') primers were used for PCR amplification and Sanger sequencing. DNA products were electrophoresed on an ABI 3730 XL DNA sequencer (Applied Biosystems, Bedford, MA).

\section{Results}

\section{WES analysis of the five patients}

Five patients with severe EOPE were recruited in this study; their clinical information and data are shown in Table 1. The incidence of EOPE was about $0.5-0.8 \%$, so the incidence of severe EOPE is much lower than EOPE. We speculated that genetic factors may play an important role in patients with severe EOPE; therefore, WES was performed for each patient. The quality of sequencing met the requirements of the bioinformatics analysis, as shown in the WES report (Supplementary Table 1).

Next, we performed a filtering process on the WES data (Supplementary Table 2). First, we retained missense, nonsense, frameshift, and splice site mutations, then filtered out the variants with allele frequencies above 1\% in the 1000G, ESP6500, ExAC, and gnomAD databases. We then used our inhouse database to further filter out duplicate mutations, and thus obtained variants for each patient. We identified 419 single nucleotide variants (SNVs) and 36 insertion-deletion mutations (InDels) in P1, 431 SNVs and 35 InDels in P2, 449 SNVs and 38 InDels in P3, 297 SNVs and 17 InDels in P4, and 398 SNVs and 31 InDels in P5 (Supplementary Table 2). All variants were further filtered according to the list of 40 selected candidate genes that are known to be associated with severe preeclampsia [9]. In this way, we were able to narrow down the scope of the target. In the five patients, we only identified a rare variant of the GOT1 gene c.44C>G:p.P15R in P1; the mutation was confirmed by Sanger sequencing (Figure $1 \mathrm{~A})$.

\section{In silico analysis of the GOT1 variant}

The Pro15 site is highly conserved across species from human to zebrafish (Figure 1B), suggesting that Pro15 plays an important role in the function of the GOT1 protein. After searching exome and genome sequencing databases, we found that the allele frequency of $c .44 \mathrm{C}>\mathrm{G}$ was 0 in all databases (Table 2), indicating that the mutation is extremely rare. Furthermore, several online mutational prediction tools predicted P15R to be a pathogenic mutation (Table 2). As the variant is heterozygous, we wanted to know whether the heterozygous variant 
influences disease tolerance. Constraint Metrics Z score for missense variation analysis [22] found that GOT1 was predicted to be intolerant to variation ( $z=2.17$, http://exac.broadinstitute.org/gene/ENSG00000120053). Thus, the above bioinformatics analysis suggests that the rare variant c.44C>G:p.P15R of the GOT1 gene may be associated with severe EOPE.

\section{Molecular modeling of the mutated protein}

Fortunately, we found that the structure of GOT1 protein had been resolved (PDB 3ii0, Figure 2A). By structure analysis, we found that there was an 'open pocket' at the surface of GOT1 protein, which was positive charged (Figure 2C). The 15th residue proline was located at the edge of this pocket (Figure 2C). In our study, this residue mutated to arginine (Figure 2B). Arginine is a basic amino acid with a big side chain (Figure 2D). We proposed that this point mutation would change the electric environment of enzymatic center, meanwhile, affect the binding affinity of substrate or product even more the whole process of enzyme-catalyzed reactions. Computer modeling had confirmed our proposal that P15R mutation changed the electricity and enhanced the positive charge of this area (Figure 2D).

\section{Discussion}

In this study, whole-exome sequencing was performed in 5 patients with severe EOPE. A rare mutation in the GOT1 gene, c.44C>G:p.P15R was found in one patient (P1). Bioinformatics analysis showed that the mutation was highly conserved across different species and was predicted to be a pathogenic mutation according to several online mutational function prediction software packages. Further structural biology homology modeling suggested that P15R may change the electricity and enhance the positive charge of this area.

The GOT1 gene-full name glutamate oxaloacetate transaminase-is also known as aspartate aminotransferase. GOT1 can catalyze Lcysteine or L-aspartate substrates. L-cysteine can be converted into 3-mercaptopyruvate by GOT1, and then $\mathrm{H}_{2} \mathrm{~S}$ can be obtained by 3mercaptopyruvate sulphurtransferase. L-aspartate can be catalyzed into L-glutamate by GOT1, which is a pyridoxal phosphate dependent enzyme found in the cytoplasm.

Previous studies suggest that maternal aspartate metabolic or catalytic processes are associated with severe preeclampsia [9]. A study using high-resolution magic angle spinning nuclear magnetic resonance spectroscopy (HR-MAS MRS) found that aspartate levels are higher in the placenta of patients with preeclampsia, while glutamate levels are lower [23]. Another study using ultra performance liquid chromatography-mass spectrometry (UPLC-MS) found that glutamate levels were low in explanted placental villous fragment cultures of preeclampsia patients [24]. The P15R mutation identified in the present study is located near the GOT1 enzyme activity center. Arginine is an alkaline amino acid, while both the substrate (L-aspartate) and product (L-glutamate) involved in GOT1 reaction are acidic. The mutated protein thus, may enhance alkalinity near the active center of the enzyme, which may increase the binding ability of the substrate or the product, and in turn affect the release of the product. Thus, the decrease in L-glutamate release due to the P15R mutation in the GOT1 gene could be related to the occurrence of preeclampsia in this patient.

A previous study found that hydrogen sulfide $\left(\mathrm{H}_{2} \mathrm{~S}\right)$ is essential for a healthy placental vasculature, and that a decrease in $\mathrm{H}_{2} \mathrm{~S}$ activity is related to the onset of preeclampsia [25]. $\mathrm{H}_{2} \mathrm{~S}$ can cause contraction and relaxation of human umbilical vein endothelial cells [26]. The formation of hydrogen sulfide is catalyzed by GOT1, which is expressed by human umbilical artery endothelial and smooth muscle cells [26]. Thus, the P15R mutation may reduce the release of 3-mercaptopyruvate, thereby affecting the production of hydrogen sulfide, which could then induce preeclampsia owing to an abnormal placental vasculature.

Previous epidemiological studies have suggested that genetic factors account for about $55-60 \%$ of PE cases, of which $30-35 \%$ can be attributed to maternal genetic factors and $20 \%$ to fetal genetic factors $[27,28,11]$. Therefore, one third of PE may be related to maternal genetic factors. In this study, we tried to find potential genetic pathogenic factors in 5 patients with severe EOPE, and the range of genetic genes was based on 40 gene panels related to severe EOPE. Only one patient with severe EOPE was found to have a potential pathogenic genetic mutation, and no other four patients were found to have a candidate pathogenic mutation. Before the study, we thought that we might find a higher proportion of genetic mutations in EOPE. But at present, only 20\% (one patient) of the genetic pathogenicity was found, which may be related to the small number of genes in the gene panel we used. We speculated that there may be more than 40 genes that can lead to severe EOPE. In the future, with the further study of PE genetics, the gene list will be more perfect, and more cases that we can find the cause will be found.

In summary, the P15G mutation in the GOT1 gene identified in the present study may lead to preeclampsia by causing abnormal synthesis of glutamate or hydrogen sulfide. Furthermore, the findings suggest that abnormal aspartate metabolic or catalytic processes, together with placental vasculature contraction or relaxation abnormalities, may be associated with preeclampsia. This study provides a genetic basis for the etiology and molecular mechanisms that underlie preeclampsia. 


\section{Declarations}

\section{Ethics approval and consent to participate}

This study was approved by the Ethics Committee of Beijing Obstetrics and Gynecology Hospital and was in accordance with the 1964 Helsinki Declaration and its later amendments. Each participant in this study signed an informed consent form to participate in this study.

\section{Consent to publish}

Each participant in this study signed an informed consent form for publication of all of the participants' clinical details.

\section{Availability of data and materials}

The datasets used and/or analysed during the current study are available from the corresponding author on reasonable request.

\section{Conflict of interest}

The authors declare no conflict of interest.

\section{Funding}

This study was supported by the Beijing Obstetrics and Gynecology Hospital, Capital Medical University [Grant No. FCYY201624].

\section{Authors' Contributions}

L.Z. carried out the majority of the experiment; L.L. performed WES data analysis and in silico analysis; F.F. performed the molecular modeling analysis; samples from the patient were collected by L.Z., Z.C., and Y.-N. X.; L.L., and H.G. designed the experiments and wrote the manuscript.

\section{Acknowledgements}

We thank PDB archive that data files contained in PDB archive are free of all copyright restrictions and made fully and freely available for both non-commercial and commercial use. We thank the deposition authors of GOT1 crystal structure (PDB ID: 3ll0), Ugochukwu, E., Pilka, E., Cooper, C., Bray, J.E., Yue, W.W., Muniz, J., Chaikuad, A., von Delft, F., Bountra, C., Arrowsmith, C.H., Weigelt, J., Edwards, A., Kavanagh, K.L., Oppermann, U., Structural Genomics Consortium (SGC). Crystal structure of human Glutamate oxaloacetate transaminase 1 (GOT1).

\section{References}

1. Zhang J, Meikle S, Trumble A. Severe maternal morbidity associated with hypertensive disorders in pregnancy in the United States. Hypertens Pregnancy. 2003;22(2):203-12.

2. Roberts JM. Preeclampsia: what we know and what we do not know. Semin Perinatol. 2000;24(1):24-8.

3. Roberts JM, Taylor RN, Musci TJ, Rodgers GM, Hubel CA, McLaughlin MK. Preeclampsia: an endothelial cell disorder. Am J Obstet Gynecol. 1989;161(5):1200-4.

4. Stillman IE, Karumanchi SA. The glomerular injury of preeclampsia. J Am Soc Nephrol. 2007;18(8):2281-4.

5. Roberts JM. Endothelial dysfunction in preeclampsia. Semin Reprod Endocrinol. 1998;16(1):5-15.

6. Redman CW, Sargent IL. Pre-eclampsia, the placenta and the maternal systemic inflammatory response--a review. Placenta. 2003;24 Suppl A:S21-7.

7. Craici IM, Wagner SJ, Weissgerber TL, Grande JP, Garovic VD. Advances in the pathophysiology of pre-eclampsia and related podocyte injury. Kidney Int. 2014;86(2):275-85.

8. Powers RW, Roberts JM, Plymire DA, Pucci D, Datwyler SA, Laird DM et al. Low placental growth factor across pregnancy identifies a subset of women with preterm preeclampsia: type 1 versus type 2 preeclampsia? Hypertension. 2012;60(1):239-46.

9. Triche EW, Uzun A, DeWan AT, Kurihara I, Liu J, Occhiogrosso R et al. Bioinformatic approach to the genetics of preeclampsia. Obstet Gynecol. 2014;123(6):1155-61.

10. Mogren I, Hogberg U, Winkvist A, Stenlund H. Familial occurrence of preeclampsia. Epidemiology. 1999;10(5):518-22.

11. Nilsson E, Salonen Ros H, Cnattingius S, Lichtenstein P. The importance of genetic and environmental effects for pre-eclampsia and gestational hypertension: a family study. BJOG. 2004;111(3):200-6. 
12. McGinnis R, Steinthorsdottir V, Williams NO, Thorleifsson G, Shooter S, Hjartardottir S et al. Variants in the fetal genome near FLT1 are associated with risk of preeclampsia. Nat Genet. 2017;49(8):1255-60.

13. Gray KJ, Saxena R, Karumanchi SA. Genetic predisposition to preeclampsia is conferred by fetal DNA variants near FLT1, a gene involved in the regulation of angiogenesis. Am J Obstet Gynecol. 2018;218(2):211-8.

14. Gray KJ, Kovacheva VP, Mirzakhani H, Bjonnes AC, Almoguera B, DeWan AT et al. Gene-Centric Analysis of Preeclampsia Identifies Maternal Association at PLEKHG1. Hypertension. 2018;72(2):408-16.

15. Reidy KJ, Hjorten RC, Simpson CL, Rosenberg AZ, Rosenblum SD, Kovesdy CP et al. Fetal-Not Maternal-APOL1 Genotype Associated with Risk for Preeclampsia in Those with African Ancestry. Am J Hum Genet. 2018;103(3):367-76.

16. Hill LD, Hilliard DD, York TP, Srinivas S, Kusanovic JP, Gomez R et al. Fetal ERAP2 variation is associated with preeclampsia in African Americans in a case-control study. BMC Med Genet. 2011;12:64.

17. Johnson MP, Roten LT, Dyer TD, East CE, Forsmo S, Blangero J et al. The ERAP2 gene is associated with preeclampsia in Australian and Norwegian populations. Hum Genet. 2009;126(5):655-66.

18. Sun CJ, Li L, Li X, Zhang WY, Liu XW. Novel SNPs of WNK1 and AKR1C3 are associated with preeclampsia. Gene. 2018;668:27-32.

19. Sun CJ, Li L, Li XY, Zhang WY, Liu XW. Associations of polymorphisms of CYP2D6 and CYP2C9 with early onset severe pre-eclampsia and response to labetalol therapy. Arch Gynecol Obstet. 2018;298(1):125-32.

20. Raymond D, Peterson E. A critical review of early-onset and late-onset preeclampsia. Obstet Gynecol Surv. 2011;66(8):497-506.

21. Bartsch E, Medcalf KE, Park AL, Ray JG. Clinical risk factors for pre-eclampsia determined in early pregnancy: systematic review and meta-analysis of large cohort studies. BMJ. 2016;353:i1753.

22. Samocha KE, Robinson EB, Sanders SJ, Stevens C, Sabo A, McGrath LM et al. A framework for the interpretation of de novo mutation in human disease. Nat Genet. 2014;46(9):944-50.

23. Austdal M, Thomsen LC, Tangeras LH, Skei B, Mathew S, Bjorge L et al. Metabolic profiles of placenta in preeclampsia using HR-MAS MRS metabolomics. Placenta. 2015;36(12):1455-62.

24. Dunn WB, Brown M, Worton SA, Crocker IP, Broadhurst D, Horgan R et al. Changes in the metabolic footprint of placental explantconditioned culture medium identifies metabolic disturbances related to hypoxia and pre-eclampsia. Placenta. 2009;30(11):974-80.

25. Wang K, Ahmad S, Cai M, Rennie J, Fujisawa T, Crispi F et al. Dysregulation of hydrogen sulfide producing enzyme cystathionine gamma-lyase contributes to maternal hypertension and placental abnormalities in preeclampsia. Circulation. 2013;127(25):2514-22.

26. Mohammed R, Provitera L, Cavallaro G, Lattuada D, Ercoli G, Mosca F et al. Vasomotor effects of hydrogen sulfide in human umbilical vessels. J Physiol Pharmacol. 2017;68(5):737-47.

27. Cnattingius S, Reilly M, Pawitan Y, Lichtenstein P. Maternal and fetal genetic factors account for most of familial aggregation of preeclampsia: a population-based Swedish cohort study. Am J Med Genet A. 2004;130A(4):365-71.

28. Esplin MS, Fausett MB, Fraser A, Kerber R, Mineau G, Carrillo J et al. Paternal and maternal components of the predisposition to preeclampsia. N Engl J Med. 2001;344(12):867-72.

\section{Tables}

Table 1. Clinical features of the patients with early-onset severe preeclampsia 


\begin{tabular}{|c|c|c|c|c|c|}
\hline Variables & $\overline{\mathrm{P} 1}$ & $\overline{\mathrm{P} 2}$ & P3 & $\overline{\mathrm{P} 4}$ & $\overline{\mathrm{P} 5}$ \\
\hline Age (years) & 35 & 27 & 29 & 31 & 31 \\
\hline Pro-gestational BMI $\left(\mathrm{kg} / \mathrm{m}^{2}\right)$ & 22.9 & 22.1 & 20 & 26.8 & 22 \\
\hline Prior pre-eclampsia & + & & & + & \\
\hline Registered gestational age (weeks) & 28 & 27 & 27 & 26 & 31 \\
\hline First birth & & + & + & + & + \\
\hline \multicolumn{6}{|l|}{ Anemia } \\
\hline Thrombocytopenia & & & + & + & \\
\hline Impaired liver function & & & + & + & + \\
\hline Progressive renal insufficiency & + & + & + & + & \\
\hline Serous membrane fluid & & + & & & + \\
\hline Pulmonary edema & & & + & & + \\
\hline Hypoproteinemia & + & & + & & - \\
\hline HELLP syndrome & & & + & + & + \\
\hline Intrahepatic cholestasis of pregnancy & & & & & + \\
\hline \multicolumn{6}{|l|}{ Placental abruption } \\
\hline Late abortion & & + & + & + & \\
\hline Premature birth & & & & & + \\
\hline Cesarean delivery & & + & + & & + \\
\hline Fetal growth restriction & + & & & & \\
\hline Stillbirth & + & & & + & \\
\hline Low birth weight infant & & + & + & & + \\
\hline
\end{tabular}

Table 2. In silico analysis of GOT1 mutation

\begin{tabular}{|c|c|c|c|c|c|c|c|c|c|c|c|}
\hline Variants & $\begin{array}{l}\text { Amino } \\
\text { acid } \\
\text { change }\end{array}$ & $\begin{array}{c}\text { Polyphen- } \\
2^{\text {a }}\end{array}$ & $\mathrm{SIFT}^{\mathrm{b}}$ & PROVEAN $^{\mathrm{C}}$ & $\begin{array}{l}\text { Mutation } \\
\text { Taster }^{\mathrm{d}}\end{array}$ & $\mathrm{SNPs} \& G O^{\mathrm{e}}$ & $\begin{array}{l}\text { FATHMM- } \\
\text { MKL }^{\mathrm{f}}\end{array}$ & gnom $A D^{g}$ & ExAC $^{h}$ & $\begin{array}{c}1000 \\
\text { Genomes }^{\mathrm{i}}\end{array}$ & ESP6500 \\
\hline c. $44 \mathrm{C}>\mathrm{G}$ & $\begin{array}{c}\mathrm{p} . \\
\text { P15R }\end{array}$ & $\begin{array}{c}\text { Possibly } \\
\text { damaging } \\
(0.924)\end{array}$ & $\begin{array}{c}\text { Damaging } \\
(0.015)\end{array}$ & $\begin{array}{c}\text { Deleterious } \\
(-7.30)\end{array}$ & $\begin{array}{c}\text { Disease } \\
\text { causing } \\
(0.9999) \\
\end{array}$ & $\begin{array}{l}\text { Disease } \\
(0.918)\end{array}$ & $\begin{array}{c}\text { Damaging } \\
(0.918)\end{array}$ & 0 & 0 & 0 & 0 \\
\hline
\end{tabular}

aPolyphen-2. Prediction Scores range from 0 to 1 with high scores indicating probably or possibly damaging.

bSIFT, i.e., Sorting Intolerant From Tolerant. Scores vary between 0 and 1 . Variants with scores close or equal to 0 are predicted to be damaging.

CPROVEAN. Variants with scores lower than -2.5 (cutoff) are predicted to be deleterious.

dMutation Taster. The probability value is the probability of the prediction, i.e., a value close to 1 indicates a high 'security' of the prediction. e SNPs\&GO. Probability: Disease probability (if $>0.5$ mutation is predicted Disease).

${ }^{\mathrm{f}}$ FATHMM-MKL. Values above 0.5 are predicted to be deleterious, while those below 0.5 are predicted to be neutral or benign.

gFrequency of variation in total of gnomAD database.

${ }^{\mathrm{h}}$ Frequency of variation in total of ExAC database.

${ }^{\mathrm{i}}$ Frequency of variation in 1000 Genomes database.

${ }^{\mathrm{j}}$ Frequency of variation in ESP6500 database.

\section{Figures}


A

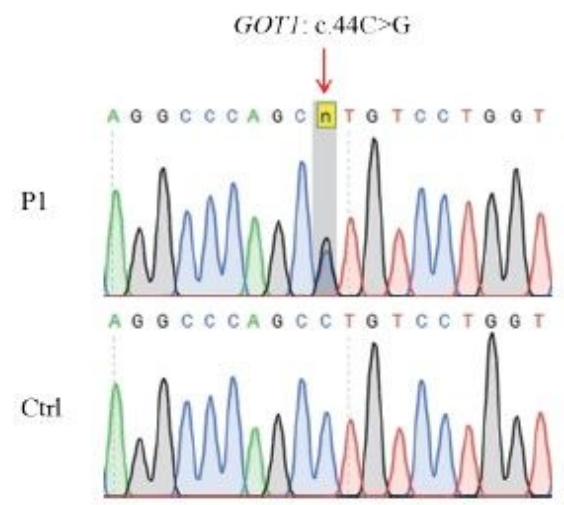

B

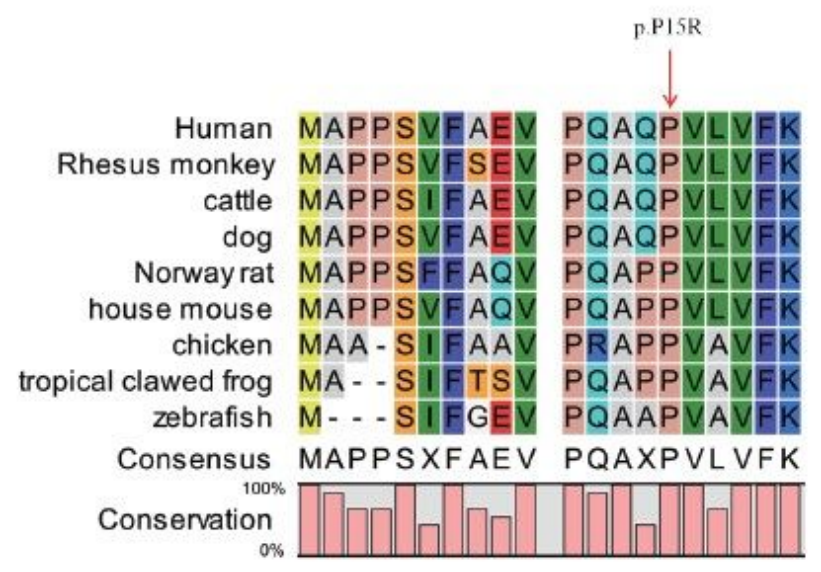

Figure 1

Analysis of the GOT1 variant. (A) Sanger sequencing validated the heterozygous $c .44 C>G$ variant in the GOT1 gene. Red arrow indicates mutation site. (B) Amino acid sequence alignment of GOT1 in different species. Red arrow indicates mutated amino acid. Proline at position 15 is $100 \%$ conserved in all species. 

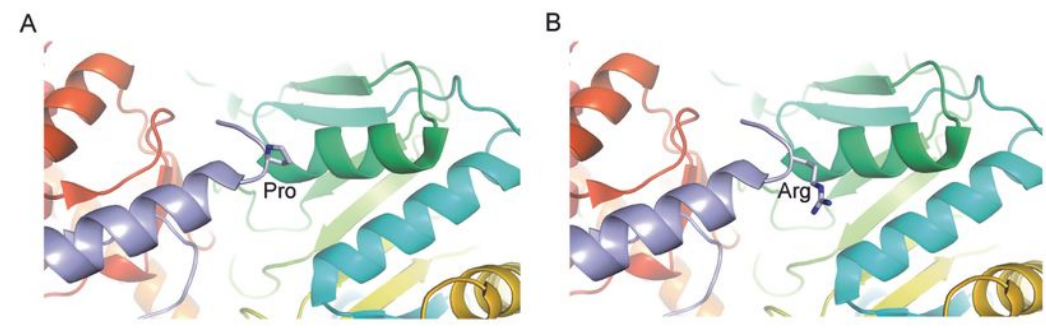

C

D
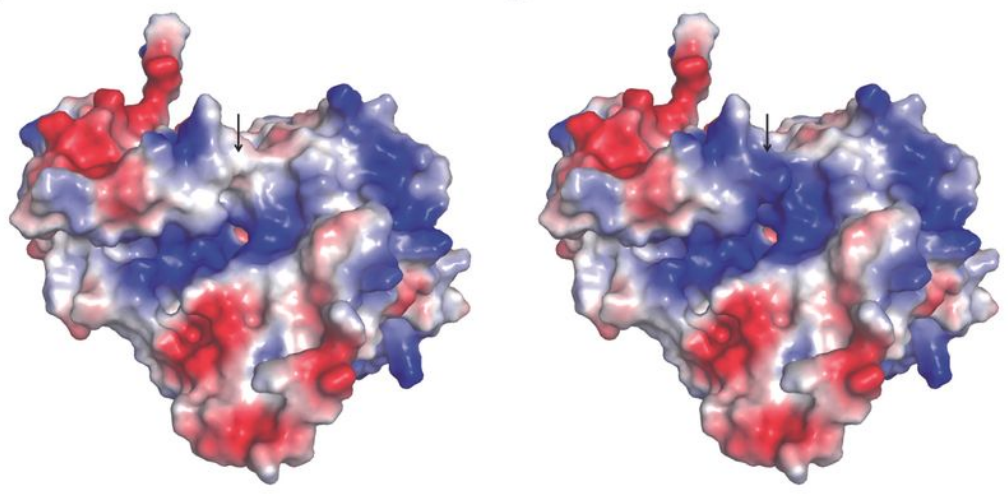

$-64$

$\mathrm{kT} / \mathrm{e}$

$+64$

\section{Figure 2}

The molecular model of GOT1 protein. (A) Close view of wild type GOT1 protein structure (PDB ID: 3II0, Ugochukwu, E., Pilka, E., Cooper, C., Bray, J.E., Yue, W.W., Muniz, J., Chaikuad, A., von Delft, F., Bountra, C., Arrowsmith, C.H., Weigelt, J., Edwards, A., Kavanagh, K.L., Oppermann, U., Structural Genomics Consortium (SGC). Crystal structure of human Glutamate oxaloacetate transaminase 1 (GOT1)). The whole protein is shown as cartoon in rainbow and 15th proline side chain is shown as sticks. (B) Close view of P15R mutant type GOT1 protein structure. The whole protein is shown as cartoon in rainbow and 15th arginine side chain is shown as sticks. (C) Electrostatic surface view of the wild type GOT1 protein. Electrostatic potential is expressed as a spectrum ranging from $-64 \mathrm{kT} / \mathrm{e}$ (red) to $+64 \mathrm{kT} / \mathrm{e}$ (blue). Black arrow indicates the 15th residue proline. (D) Electrostatic surface view of the P15R mutation GOT1 protein. Electrostatic potential is expressed as a spectrum ranging from $-64 \mathrm{kT} / \mathrm{e}$ (red) to $+64 \mathrm{kT} / \mathrm{e}$ (blue). Black arrow indicates the 15 th residue arginine.

\section{Supplementary Files}

This is a list of supplementary files associated with this preprint. Click to download.

- SuppTables.pdf 